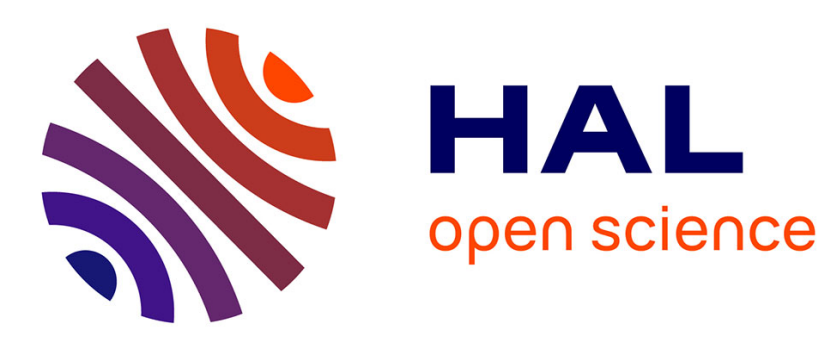

\title{
Transversely polarized parton densities their evolution and their measurement
}

\author{
X. Artru, M. Mekhfi
}

\section{To cite this version:}

X. Artru, M. Mekhfi. Transversely polarized parton densities their evolution and their measurement. Zeitschrift für Physik C Particles and Fields, 1990, 45, pp.669-676. 10.1007/BF01556280 . in2p300001002

\section{HAL Id: in2p3-00001002 \\ https://hal.in2p3.fr/in2p3-00001002}

Submitted on 28 Jun 2021

HAL is a multi-disciplinary open access archive for the deposit and dissemination of scientific research documents, whether they are published or not. The documents may come from teaching and research institutions in France or abroad, or from public or private research centers.
L'archive ouverte pluridisciplinaire HAL, est destinée au dépôt et à la diffusion de documents scientifiques de niveau recherche, publiés ou non, émanant des établissements d'enseignement et de recherche français ou étrangers, des laboratoires publics ou privés. 


\title{
Transversely polarized parton densities, their evolution and their measurement
}

\author{
X. Artru ${ }^{1}$ and M. Mekhfi ${ }^{2}$ \\ ${ }^{1}$ Laboratoire de Physique Théorique et Hautes Energies`, Université de Paris XI, Bâtiment 211, F-91405 Orsay, France \\ 2 Laboratoire de Physique Théorique, Université d’Oran Es-Senia, Oran Es-Senia, 31, Algéria
}

\begin{abstract}
The transverse spin asymmetry of a quark in a baryon and the linear polarization of a gluon in a vector meson are studied from the $t$-channel point of view. Using the Altarelli-Parisi approach, they are shown to obey independent evolution equations and to decrease with increasing $Q^{2}$. We investigate the possibility to measure them at leading twist, to leading order in $\alpha$ and $\alpha_{s}$ and without analyzing the final polarizations. This requires simultaneous polarization of the beam and the target; the observable effect is in the azimuthal distribution of the high $P_{T}$ particle or jet. Assuming a simple (quark + scalar diquark) model for the baryon, a large asymmetry is expected in $p \bar{p}$ Drell-Yan collisions, a smaller one in high $P_{T} p p$ collisions, from the interference term in the scattering of two identical quarks.
\end{abstract}

\section{Introduction}

In the parton model [1-3], the relevant quark and gluon densities for polarized beam or target experiments are the following ones:

(i) $a(x)=$ unpolarized density of parton $a \cdot a=$ quark $(q)$ or gluon $(G) . x$ is the Bjorken variable; the dependence in $Q^{2}$ is understood.

(ii) $\Delta a(x)=a_{+}(x)-a_{-}(x)$, where $a_{+}(x)$ and $a_{-}(x)$ are respectively the densities of parton $a$ of positive and negative helicity, when the hadron has positive helicity. (iii) $\Delta_{1} q(x)=q_{A}(x)-q_{-A}(x)$, where $q_{ \pm n}(x)$ is the density of quarks polarized in the transverse direction $\pm \hat{n}$, when the hadron spin points in the direction $+\hat{n}$.

(iv) $\Delta_{2} G(x)=G_{x}(x)-G_{\hat{\vartheta}}(y)$, where $G_{\hat{n}}(x)(\hat{n}=\hat{x}$ or $\hat{y})$ is the density of gluon linearly polarized along $\hat{n}$,

\footnotetext{
* Laboratoire associé au Centre National de la Recherche Scientifique
}

the hadron being a vector meson linearly polarized along $\hat{x}$.

We have obviously

$$
\begin{aligned}
q(x) & =q_{+}(x)+q_{-}(x)=q_{\hat{n}}(x)+q_{-\hat{n}}(x) \\
G(x) & =G_{+}(x)+G_{-}(x)=G_{\hat{x}}(x)+G_{\hat{\jmath}}(x) .
\end{aligned}
$$

Whereas a lot of theoretical and experimental work has been devoted to $a(x)$ and $\Delta a(x)$, the transverse polarization asymmetries $\Delta_{1} q(x)$ and $\Delta_{2} G(x)$ have not been, up to now, popular topics. A good reason for this is that they are not easy to measure; we shall discuss this question is Sect. 4. But, in the case of $\Delta_{1} q(x)$, there is also the prejudice that it vanishes in the limit of zero mass and zero transverse momentum of the quark. This is totally unjustified; in the case of electrons, for instance, the smallness of the electron mass does not preclude large transverse polarizations of ultrarelativistic electrons; the effect of such polarization is well known in $e^{+} e^{-}$collisions [4]. To show that $\Delta_{1} q(x)$ is not necessarily vanishing, we present, in Appendix $C$, a naïve covariant parton model where the baryon is composed of a quark and a scalar diquark. It predicts

$\Delta_{1} q(x)=q_{+}(x)$.

Concerning the evolution of $\Delta_{1} q(x)$, previous theoretical works have yielded different results [5]. As for $\Delta_{2} G(x)$, we have not found any explicit mention of this quantity in the literature. It is involved, however, implicitly in the polarized photon structure function [6].

For the above reasons, we think it worthwhile to derive independently the evolution equations of the transverse asymmetries, using the Altarelli-Parisi approach, and to discuss their observable effects at leading twist and to lowest order in $\alpha$ and $\alpha_{s}$. These problems will be more easily treated in the $t$-channel formalism which we have already introduced to handle spin in multiparton reactions [7]. 
This work will be developed as follows. In the next subsection, we present the $t$-channel formalism for the single parton distributions. In Sect. 3, we derive the evolution equations of the transversely polarized parton distributions. We discuss the observability of these distributions in Sect. 4. Conclusions are presented in Sect. 5 .

\section{The $t$-channel spin formalism for parton distributions}

In a recent work [7], we have set up a quite general and straightforward formalism to handle spin in single- and multiparton scattering, based on the $t$ channel analysis of the helicity amplitude. Consider the hard collision whose unitarity diagram is drawn in Fig. 1. The cross section for producing a particular hard final state $f$ is

$$
\begin{aligned}
\sigma= & \sum_{\text {all } \lambda^{\prime} s} \int d x\left\langle\lambda_{a}\left|\rho^{a}(x)\right| \lambda_{a}^{\prime}\right\rangle \int d y\left\langle\lambda_{b}\left|\rho^{b}(y)\right| \lambda_{b}^{\prime}\right\rangle \\
& \cdot\left\langle\lambda_{a}^{\prime}, \lambda_{b}^{\prime}\left|H^{a+b \rightarrow f}\right| \lambda_{a}, \lambda_{b}\right\rangle,
\end{aligned}
$$

where $x=p_{a} / p_{A}, y=p_{b} / p_{B}$,

$$
\begin{aligned}
& \left\langle\lambda_{a}^{\prime}, \lambda_{b}^{\prime}\left|H^{a+b \rightarrow f}\right| \lambda_{a}, \lambda_{b}\right\rangle \\
& \quad \approx\left\langle a, \lambda_{a}^{\prime} ; b, \lambda_{b}^{\prime}\left|T^{+}\right| f\right\rangle\left\langle f|T| a, \lambda_{a} ; b, \lambda_{b}\right\rangle,
\end{aligned}
$$

is a partial discontinuity of the forward parton-parton amplitude, $\rho^{a}(x)$ is a density matrix in spin space, related to the hadronic density matrix $\rho^{A}$ by

$$
\begin{aligned}
& \left\langle\lambda_{a}\left|\rho^{a}(x)\right| \lambda_{a}^{\prime}\right\rangle \\
& \quad=\sum_{\lambda_{A}^{\prime}, \lambda_{A}}\left\langle\lambda_{A}^{\prime}, \lambda_{a}\left|\Gamma^{a / A}(x)\right| \lambda_{A}, \lambda_{a}^{\prime}\right\rangle\left\langle\lambda_{A}\left|\rho^{A}\right| \lambda_{A}^{\prime}\right\rangle,
\end{aligned}
$$

and similarly for $\rho^{b}(y) . \Gamma^{a / A}(x)$ is the hadron-parton cut amplitude; unlike $\rho^{a}(x)$ it does not depend on the actual hadron polarization.

For each particle-antiparticle pair in the $t$-channel of Fig. 1, we define the $t$-channel helicity state to be simply

$$
|\lambda\rangle \otimes\left|\bar{\lambda}^{\prime}\right\rangle \text {, }
$$

where

$\bar{\lambda}^{\prime} \equiv-\lambda^{\prime}$.

Then we build a basis of particle-antiparticle helicity states $|\Lambda\rangle$ which have definite total helicity

$\delta=\lambda+\bar{\lambda}^{\prime}=\lambda-\lambda^{\prime}$,

and definite symmetry $\varepsilon= \pm$. For particles of spin $s$ which can only take two possible helicities, $+s$ or $-s$, the $|\boldsymbol{\Lambda}\rangle$ states are:

$$
\begin{array}{r}
\left|0_{+}\right\rangle=\frac{1}{\sqrt{2}}(|s\rangle \otimes|-s\rangle+|-s\rangle \otimes|s\rangle) \\
(\delta=0, \varepsilon=+) \\
\left|0_{-}\right\rangle=\frac{1}{\sqrt{2}}(|s\rangle \otimes|-s\rangle-|-s\rangle \otimes|s\rangle) \\
(\delta=0, \varepsilon=-)
\end{array}
$$

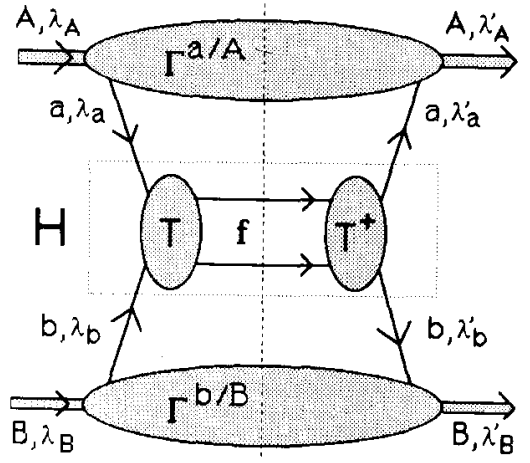

Fig. 1. Unitarity diagram for a hard hadron-hadron collision with the subprocess $a+b \rightarrow f$

$$
\begin{array}{r}
|2 s\rangle=|s\rangle \otimes|s\rangle ; \quad|-2 s\rangle=|-s\rangle \otimes|-s\rangle \\
(\delta= \pm 2 s, \varepsilon=+) .
\end{array}
$$

Let us first consider the " $t$-channel amplitude" $\tilde{\Gamma}^{a / A}(x)$ obtained by crossing $\Gamma^{a / A}(x)$ and sandwiching it between $|\Lambda\rangle$ states. Due to rotational and parity invariance, $\tilde{\Gamma}^{a / A}(x)$ conserves $\delta$ and $\varepsilon$, i.e.

$\delta_{a}=\lambda_{a}-\lambda_{a}^{\prime}=\delta_{A}=\lambda_{A}-\lambda_{A}^{\prime}$

$\varepsilon_{a}=\varepsilon_{A}$,

so we can write

$$
\left\langle\Lambda_{a}\left|\tilde{\Gamma}^{a / A}(x)\right| \Lambda_{A}\right\rangle=\delta_{A_{a}, \Lambda_{A}} \tilde{\Gamma}_{\Lambda_{a}}^{a / A}(x) .
$$

It turns out that the $\tilde{\Gamma}_{\Lambda}^{a / A}(x)$ are just equal to the parton distributions defined in Sect. 1:

$$
\begin{aligned}
a(x) & =\tilde{\Gamma}_{0_{+}}(x), \\
\Delta a(x) & =\tilde{\Gamma}_{0_{-}}(x), \\
\Delta_{1} q(x) & =\tilde{\Gamma}_{1}(x)=\tilde{\Gamma}_{-1}(x), \\
\Delta_{2} G(x) & =\tilde{\Gamma}_{2}(x)=\tilde{\Gamma}_{-2}(x) .
\end{aligned}
$$

As expected, transverse polarization asymmetries are associated with non-zero total helicity $\delta$ in the $t$-channel, i.e., helicity flip in the $s$-channel. Due to the conservation of $\delta$ and $\varepsilon$, we can already predict that $\Delta_{1} q$ and $\Delta_{2} G$ satisfy simple (unmixed) evolution equations, contraryly to what happens for $\Delta q$ and $\Delta G[2]$.

As we have done for $\Gamma$, we can similarly decompose the matrix densities $\rho$ and the parton-parton discontinuity $H$ in the $t$-channel basis. For this purpose, we prefer to reformulate the $t$-channel analysis in a more physical way:

To each $t$-channel state

$$
|\Lambda\rangle=\sum c_{\lambda \mu}|\lambda\rangle \otimes|\mu\rangle \text {, }
$$

defined in (2.6), we associate the $s$-channel operator

$\mathcal{O}(\Lambda)=\sum_{\lambda, \mu} c_{\lambda \mu}|\lambda\rangle\langle-\mu|$ 
Thus

$$
\begin{aligned}
& \mathcal{O}\left(0_{+}\right)=\frac{1}{\sqrt{2}} 1, \\
& \mathcal{O}\left(0_{-}\right)=\frac{1}{\sqrt{2}}\left(\begin{array}{cc}
1 & 0 \\
0 & -1
\end{array}\right)=\frac{1}{\sqrt{2}} \sigma_{z}, \\
& \mathcal{O}( \pm 2 s)=| \pm s\rangle\langle\mp s|=\sigma_{ \pm} \equiv \frac{1}{2}\left(\sigma_{x} \pm i \sigma_{y}\right) \text {. }
\end{aligned}
$$

The orthogonality of the $|\Lambda\rangle$ states yields

$\operatorname{tr}\left[\mathcal{O}(\Lambda) \mathcal{O}^{+}\left(\Lambda^{\prime}\right)\right]=\delta_{\Lambda \Lambda^{\prime}}$.

Then we decompose $\rho^{A}, \rho^{B}, \rho^{a}(x), \rho^{b}(y), \Gamma^{a / A}(x), \Gamma^{b / B}(y)$ and $H$ on the $s$-channel operators $\mathcal{O}(\Lambda)$ [8]:

$$
\begin{aligned}
\rho & =\sum_{\Lambda} \rho_{\Lambda} \mathcal{O}(\Lambda), \\
\Gamma^{a / A}(x) & =\sum_{\Lambda} \tilde{\Gamma}_{\Lambda}^{a / A}(x) \mathcal{O}^{+}(\Lambda)_{A} \otimes \mathcal{O}(\Lambda)_{a}, \\
H & =\sum_{\Lambda_{a}} \sum_{\Lambda_{b}} H_{\Lambda_{a} \Lambda_{b}} \mathcal{O}^{+}\left(\Lambda_{a}\right) \otimes \mathcal{O}^{+}\left(\Lambda_{b}\right) .
\end{aligned}
$$

The inverse formulas are obtained by use of (2.12); for instance

$\rho_{\Lambda}=\operatorname{tr}\left[\rho \mathcal{O}^{+}(\Lambda)\right]$.

The coefficients $\tilde{\Gamma}_{\Lambda}^{a / A}(x), H_{\Lambda_{a} \Lambda_{b}}$ are just the $t$-channel amplitudes taken between the $|\Lambda\rangle$ states. In fact we could have introduced the $\mathcal{O}(\Lambda)$ 's directly by (2.11), without any reference to $t$-channel states. This provides an alternative presentation of the formalism. We are now able to rewrite (2.1) and (2.3) as

$\sigma=\sum_{\Lambda_{a} \Lambda_{b}} \int d x \rho_{\Lambda_{a}}^{o}(x) \int d y \rho_{\Lambda_{b}}^{b}(y) H_{\Lambda_{a} \lambda_{b}}^{a+b \rightarrow f}$,

$\rho_{\Lambda}^{a}(x)=\rho_{\Lambda}^{A} \tilde{\Gamma}_{\Lambda}^{a / A}(x) \quad\left(\right.$ idem for $\left.\rho_{\Lambda}^{b}(y)\right)$.

The "unpolarized" quantities are

$\rho_{0_{+}}^{A}=\frac{1}{\sqrt{2}} ; \quad \tilde{\Gamma}_{0_{+}}^{a / A}(x)=a(x) ; \quad \rho_{0_{+}}^{a}(x)=\frac{1}{\sqrt{2}} a(x)$,

$H_{0+0+}^{a+b \rightarrow f}=2 \hat{\sigma}_{\text {unpolarized }}^{a+b \rightarrow f}$.

We give below the values of $\rho_{\Lambda} / \rho_{0+}$ corresponding to different polarization cases.

- partial helicity polarization

$\rho_{0_{-}} / \rho_{0_{+}}=\operatorname{sign}\left(p_{z}\right)\left\langle s_{z}\right\rangle / s ; \quad \rho_{ \pm 2 s}=0$,

- partial transverse polarization: $\rho_{0_{-}}=0$,

$\rho_{ \pm 2 s} / \rho_{0_{+}} \begin{cases}=\frac{P}{\sqrt{2}} e^{\mp 2 s i \psi} & \text { (forward going particle) } \\ =\frac{P}{\sqrt{2}} e^{\mp 2 s i(\pi-\downarrow)} & \text { (backward going particle [10]), }\end{cases}$

where $P \leqq 1$ is the degree of polarization and $\psi$ the azimuthal angle of the polarization direction (spinorial for $s=\frac{1}{2}$, linear for $s=1$ ).

\section{Evolution of $\Delta_{1} q(x)$ and $\Delta_{2} G(x)$}

In the physical gauge, the evolution of $\rho^{a}\left(x, Q^{2}\right)$ or, equivalently, of $\Gamma^{a / A}\left(x, Q^{2}\right)$ is given by the ladder of Fig. 2 [3]. The kernel, represented in Fig. 3, is proportional to the Altarelli-Parisi splitting matrix

$$
\begin{aligned}
& \left\langle\lambda^{\prime} \mu|\mathbb{P}(z)| \lambda, \mu^{\prime}\right\rangle \\
& \underset{z \neq 1}{=} \frac{z(1-z)}{2 k_{T}^{2}} \sum_{\beta, \gamma, v}\langle\mathbf{b}, \beta, \mu ; \mathbf{c}, \gamma, v|V| \mathbf{a}, \alpha, \lambda\rangle \\
& \cdot\left\langle\mathbf{b}, \beta, \mu^{\prime} ; \mathbf{c}, \gamma, \nu|V| \mathbf{a}, \alpha, \lambda^{\prime}\right\rangle^{*},
\end{aligned}
$$

where $\alpha, \beta, \gamma$ are color indices, $\mu, \nu, \lambda$ are helicities, $\mathbf{b}=z \mathbf{a}+\mathbf{k}_{T}$ and $\mathbf{c}=(1-z) \mathbf{a}-\mathbf{k}_{T}$. For $\Delta_{1} q(x)$ and $\Delta_{2} G(x)$, we have $\lambda=\mu=-\lambda^{\prime}=-\mu^{\prime}$. The corresponding element $\Delta_{1 \text { or } 2} P(z)=\langle-\lambda, \lambda|\mathbb{P}(z)| \lambda,-\lambda\rangle$ appears then as an interference term between two $V$ 's (at least in the helicity basis). Alternatively, we have:

$$
\begin{aligned}
& \Delta_{1} P(z)=P_{q_{+A G_{+}}}(z)-P_{q_{-A} q_{+A}}(z), \\
& \Delta_{1} P(z)=P_{G_{x} G_{x}}(z)-P_{G_{x} G_{y}}(z) .
\end{aligned}
$$

The detailed calculation of the transverse splitting functions is given in Appendix B and C. We give here just the result [11]:

$$
\begin{aligned}
& \Delta_{1} P(z)=C_{2}(R)\left[\frac{2}{(1-z)_{+}}-2+\frac{3}{2} \delta(z-1)\right], \\
& \Delta_{2} P(z) \\
& \quad=C_{2}(G)\left[\frac{2}{(1-z)_{+}}-2+\left(\frac{11}{6}-\frac{2}{3} \frac{T(R)}{C_{2}(G)}\right) \delta(z-1)\right]
\end{aligned}
$$

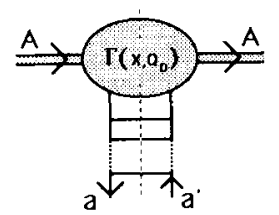

Fig. 2. Ladder diagram describing the evolution of the parton density, in the planar gauge

(a)

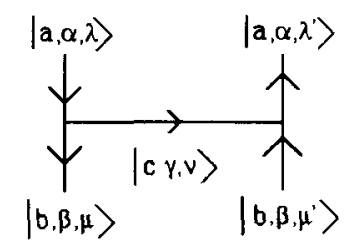

(b)

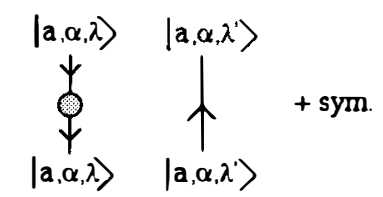

Fig. 3. Kernel of the evolution equation for the parton density matrix: a radiation of a spectator parton, b renormalization of the parton propagators 
where $C_{2}(R)=\left(N^{2}-1\right) / 2 N=4 / 3, C_{2}(G)=N=3$ and $T(R)=\frac{1}{2} N_{\text {flavor }}$. The distribution $1 /(1-z)_{+}$is defined by

$\int_{0}^{1} d z \frac{f(z)}{(1-z)_{+}}=\int_{0}^{1} d z \frac{f(z)-f(1)}{1-z}$,

$f(z)$ being any test function which is sufficiently regular at the end points.

For the quark transverse asymmetry, the master equation reads

$\frac{d}{d \ln Q^{2}} \Delta_{1} q\left(x, Q^{2}\right)=\frac{\alpha_{s}\left(Q^{2}\right)}{2 \pi} \int_{x}^{1} \frac{d y}{y} \Delta_{1} P\left(\frac{x}{y}\right) \Delta_{1} q\left(y, Q^{2}\right)$,

where $\alpha_{s}\left(Q^{2}\right)=g^{2} / 4 \pi$ is the running coupling constant. In terms of the moments

$$
\begin{aligned}
\Delta_{1} q_{n}\left(Q^{2}\right) & =\int_{0}^{1} d x x^{n-1} \Delta_{1} q\left(x, Q^{2}\right) ; \\
\Delta_{1} P_{n} & =\int_{0}^{1} d z z^{n-1} \Delta_{1} P(z),
\end{aligned}
$$

the solution of (3.4) is given by

$\Delta_{1} q_{n}\left(Q^{2}\right)=\Delta_{1} q_{n}\left(Q_{0}^{2}\right) \exp \left[\Delta_{1} P_{n} \int_{Q_{0}^{2}}^{Q^{2}} \frac{\alpha_{s}\left(Q^{\prime 2}\right)}{2 \pi Q^{\prime 2}} d Q^{\prime 2}\right]$.

In the leading logarithm approximation, we have

$\alpha\left(Q^{2}\right)=\frac{1}{b t}, \quad t=\ln \left(Q^{2} / \Lambda^{2}\right), \quad b=\frac{33-2 N_{f}}{12 \pi}$,

and we can write the exponential factor of (3.5) as

$\left(\frac{t}{t_{0}}\right) \exp \left(\frac{\Delta_{1} P_{n}}{2 \pi b}\right)$.

The moments of $\Delta_{1} P(z)$ are

$\Delta_{1} P_{n}=C_{2}(R)\left(\frac{3}{2}-2 \sum_{j=1}^{n} \frac{1}{j}\right)$.

For the gluon transverse asymmetry, we have just to replace $\Delta_{1}$ by $\Delta_{2}$ and $q$ by $G$ in $(3.4-3.7)$. We have now

$\Delta_{2} P_{n}=C_{2}(G)\left(\frac{11}{6 n}-2 \sum_{j=1}^{n} \frac{1}{j}\right)-\frac{2}{3} T(R)$.

We note that, for any $n, \Delta_{1} P_{n}$ and $\Delta_{2} P_{n}$ are negative. This means that the transverse asymmetries decrease with increasing $Q^{2}$, whereas the longitudinal spin asymmetry [12] is constant for the quark and growing for the gluon.

\section{Observability of the transverse asymmetries}

While there is no reason to assume that the transverse polarization of, say, a proton is not transmitted to its quarks (for instance, recall (1.2)), it sometimes happens that the hard process is insensitive to such a quark transverse polarization, at least to zeroth order in $\left(m_{q} / Q\right),\left(p_{T}(q) / Q\right)$ and to lowest order in $\alpha$ and $\alpha_{s}$. This is the case in deep inelastic lepton scattering, where helicity conservation at the quark photon vertex selects only the $\delta \equiv \lambda-\lambda^{\prime}=0$ components of the quark density matrix, whereas transverse polarization lies in the $\delta= \pm 1$ components. To observe transverse polarization at leading twist and to lowest order in $\alpha$ and $\alpha_{s}$, we have therefore to look at other hard processes.

Restricting ourself to $2 \rightarrow 2$ collisions, we have the following subprocesses at our disposal
a) lepton $+q \rightarrow$ lepton $+q$
b) $\quad q q \rightarrow q q$
c) $\quad q \bar{q} \rightarrow \ell \bar{\ell}, q \bar{q}, G G, G \gamma$ or $\gamma \gamma$
d) $G q$ or $\gamma q \rightarrow G q$ or $\gamma q$
e) $\quad G G \rightarrow G G$
f) $G G$ or $\gamma G \rightarrow q \bar{q}$.

In this work, we shall assume that the polarizations of the final particles are not measured. Since the final angular distribution may depend on the initial polarizations, we specify the polar angle $\theta$ and azimuthal angle $\varphi$ of the relative momentum in $f$ and write

$H^{a+b \rightarrow \mathcal{f}}=H(\theta, \varphi)$,

the $\hat{s}$ dependence being understood. Conservation of angular momentum about $\mathrm{Oz}$ gives

$\left\langle\lambda_{a}^{\prime}, \lambda_{b}^{\prime}|H(\theta, \varphi)| \lambda_{a}, \lambda_{b}\right\rangle=e^{i \varphi\left(\delta_{a}-\delta_{b}\right)}\left\langle\lambda_{a}^{\prime}, \lambda_{b}^{\prime}|H(\theta, 0)| \lambda_{a}, \lambda_{b}\right\rangle$

with $\delta=\lambda-\lambda^{\prime}$; equivalently,

$H_{\Lambda_{a} \Lambda_{b}}(\theta, \varphi)=e^{i \varphi\left(\delta_{a}-\delta_{b}\right)} H_{\Lambda_{a} \Lambda_{b}}(\theta, 0)$.

We write down other symmetry properties of $H_{A_{a} A_{b}}$ which are relevant in polarization effects (in what follows, $|\bar{\Lambda}\rangle$ is the state obtained from $|\Lambda\rangle$ by reversing $\delta$ but not $\varepsilon$ ).

-hermiticity

$H_{\Lambda_{a} \Lambda_{b}}(\theta, \varphi)=H_{\bar{\lambda}_{a} \bar{\lambda}_{b}}^{*}(\theta, \varphi)$,

- parity invariance (not necessarily true)

$H_{A_{a} \Lambda_{b}}(\theta, \varphi)=(-)^{\delta_{a}-\delta_{b}} \varepsilon_{a} \varepsilon_{b} H_{\bar{\Lambda}_{a} \bar{\Lambda}_{b}}(\theta,-\varphi)$,

$-T$ invariance + Born approximation (+ hermiticity)

$H_{A_{a} A_{b}}(\theta, \varphi)=H_{\Lambda_{a} A_{b}}^{*}(\theta,-\varphi)$,

- chirality invariance

$\delta_{a}+\delta_{b}=0 \quad(q \bar{q}, q q, \bar{q} \bar{q})$

$\delta_{a}=0 \quad(a=$ quark; $b=$ gluon or photon $)$

$\delta_{a}=\delta_{b}=0 \quad(a=$ quark; $b=$ lepton $)$

Finally, it has been shown [13] that the Born amplitudes for the $2 \rightarrow 2$ processes $(4.1 \mathrm{c}-\mathrm{f}$ ) also conserve the total helicity, in the massless limit, although no simple proof has been given for it. Then, $\lambda_{a}+\lambda_{b}$ and 
$\lambda_{a}^{\prime}+\lambda_{b}^{\prime}$ are both equal to the total helicity of the intermediate state $f$ in (2.2), hence

$\delta_{a}+\delta_{b}=0 \quad$ (to leading order in $\alpha, \alpha_{s}$ and $m / Q$ )

From (4.3-4.9) we can draw the following conclusions:

- if we integrate over $\varphi$, the only observable asymmetries (i.e., $\hat{\sigma}_{\text {polarized }} \neq \hat{\sigma}_{\text {unpolarized }}$ ) are characterized by

$\delta_{a}=\delta_{b}$.

This is the case for helicity asymmetries $\left(\delta_{a}=\delta_{b}=0\right)$. By constrast, in $q q$ or $q \bar{q}$ scattering we have no net transverse polarization effect after $\varphi$ integration (the null theorem of [4b]). The same situation holds in $G G$ or $G \gamma$ scattering to leading order in $\alpha$ and $\alpha_{s}$.

We therefore consider experiments where the data are not integrated over $\varphi$. Furthermore we assume parity conservation in the hard process [14].

\section{a) Transversely polarized quarks}

It follows immediately from (4.8) that if $a$ is a transversely polarized quark $\left(\delta_{a}= \pm 1\right), b$ must also be a transversely polarized quark or antiquark. This selects processes (4.1 $\mathrm{b}$ and $\mathrm{c})$. Then, gathering (2.9), (2.15), (2.16), (2.20), (4.4), (4.5) and (4.6),

$$
\begin{aligned}
\sigma= & \int d x d y \hat{\sigma}_{\text {unpolarized }}\left[a(x) b(y)-P_{A} P_{B} \Delta_{1} a(x) \Delta_{1} b(y)\right. \\
& \left.\cdot \hat{a}_{N N}(\theta) \cos \left(2 \varphi-\psi_{A}-\psi_{B}\right)\right] \quad(a, b=q \text { or } \bar{q}),
\end{aligned}
$$

where $P_{A}$ and $\psi_{A}$ are respectively the magnitude and azimuthal angle of the polarization vector of hadron $A$ (similarly for $B$ ) [15], $\theta$ is the scattering angle in the parton-parton center-of-mass and

$\hat{a}_{N N}(\theta)=H_{1,-1}(\theta, 0) / H_{0+0+}(\theta, 0)$,

is the transverse asymmetry parameter of the hard subprocess. In terms of $s$-channel helicities,

$$
\begin{aligned}
H_{0_{+} 0_{+}} & =\langle++|H|++\rangle+\langle+-|H|+-\rangle \\
H_{1,-1} & =\langle-+|H|+-\rangle .
\end{aligned}
$$

Due to helicity conservation, a nonvanishing $H_{1,-1}$ can only arise from $q \bar{q}$ fusion diagrams (Fig. $4 a$ ) or interference diagrams in identical $q q$ scattering (Fig. 4b). Thus a first place where transversely polarized quark distributions can be measured is Drell-Yan pair production with polarized beam and target. Here [16],

$\hat{a}_{N N}(\theta)=-\sin ^{2} \theta /\left(1+\cos ^{2} \theta\right)$.

If relation (1.2) has some part of truth, the effect is expected to be large in $p \bar{p}$ Drell-Yan collisions, but small in $p p$ ones, the polarization of the sea being probably small. In the reaction

$p p \rightarrow 2$ high $p_{T}$ jets + anything,

the interference diagram of $u u$ or $d d$ scattering (which is suppressed by a color factor in $1 / N$ ) can lead to a few percent asymmetry [17] at large $x_{T}=p_{T} / \sqrt{s}$; in this case [19]

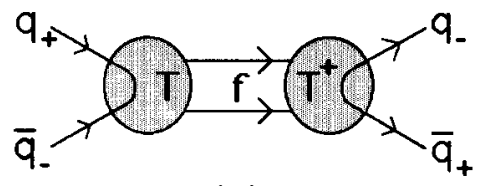

(a)

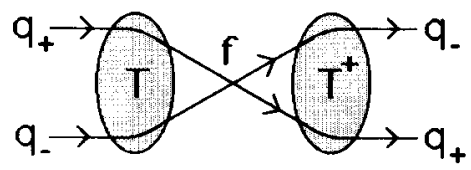

(b)

Fig. 4. Subprocesses allowing helicity flip $\delta_{a}=-\delta_{b}= \pm 1$ : a quarkantiquark annihilation, b interference term in identical quark-quark scattering

$\hat{a}_{N N}(\theta)=-\sin ^{4} \theta /\left(11+34 \cos ^{2} \theta+3 \cos ^{4} \theta\right)$.

Note the very fast decrease of $\hat{a}_{N N}(\theta)$ when we leave the $\theta=\pi / 2$ region.

\section{b) Linearly polarized gluon}

Here again, due to (4.9), both the beam and the target must be linearly polarized, which is not an easy task. This selects processes $(4.1 \mathrm{e}, \mathrm{f})$. Then, in complete analogy with (4.11) and (4.12), we have for $G G$ collisions

$$
\begin{aligned}
\sigma= & \int d x d y \hat{\sigma}_{\text {unpolarized }}\left[G(x) G(y)+P_{A} P_{B} \Delta_{2} G(x) \Delta_{2} G(y)\right. \\
& \left.\cdot \hat{a}_{\text {lin }}(\theta) \cos \left(4 \varphi-2 \psi_{A}-2 \psi_{B}\right)\right],
\end{aligned}
$$

where $P_{A}$ and $\psi_{A}$ are respectively the magnitude and azimuthal angle of the linear polarization of $A$ (idem for $B)[15]$ and

$$
\begin{aligned}
\hat{a}_{\text {lin }}(\theta) & =H_{2,-2}(\theta, 0) / H_{0_{+} 0_{+}}(\theta, 0), \\
H_{0+0+} & =\langle++|H|++\rangle+\langle+-|H|+-\rangle, \\
H_{2,-2} & =\langle-+|H|+-\rangle .
\end{aligned}
$$

(4.16) and (4.17) also apply to $\gamma G$ subprocess with the following substitutions

$G(x)$ and $\Delta_{2} G(x) \rightarrow \delta(x-1)$.

\section{Conclusion}

The transversely polarized quark distribution $\Delta_{1} q(x)$ and the linearly polarized gluon distribution $\Delta_{2} G(x)$, which correspond to non zero helicities in the $t$ channel, obey simple, uncoupled evolution equations. Their moments decrease for all $n$ as negative powers of $\ln Q^{2}$, unlike the helicity asymmetries for $n=1$.

$\Delta_{1} q(x)$ and $\Delta_{2} G(x)$ should a priori exist even in the limit of vanishing parton mass and transverse momentum. For instance, a naïve quark + scalar diquark model gave us

$\Delta_{1} q(x)=q_{+}(x)$ 
this, combined with the experimental indication [23] that

$\int q_{+}(x) d x \simeq \int q_{-}(x) d x$,

would imply

$\int \Delta_{1} q(x) d x \simeq \frac{1}{2} \int q(x) d x$.

$\Delta_{1} q(x)$ and $\Delta_{2} G(x)$ can be measured at leading twist and to leading order in $\alpha$ and $\alpha_{s}$, provided both the beam and the target are polarized (transverse spin + transverse spin for $\Delta_{1} q$, linear polarization + linear polarization for $\Delta_{2} G$ ). There is no effect in the total cross section but in the azimuthal distribution. Another method is to analyse one final polarization, in which case only one incoming polarization is needed. We did not study this possibility in the present work.

The principle of the measurement of $\Delta_{1} q(x)$ is the same as for the asymmetry parameter $a_{N N}$ in elastic scattering of two spin $\frac{1}{2}$ particle; from (4.11) we have an effective asymmetry parameter given by

$a_{N N}^{\text {eff }}=\hat{a}_{N N} \frac{\Delta_{1} a(x) \Delta_{1} b(y)}{a(x) b(y)}$,

where $x$ and $y$ are the fractional momenta of colliding partons $a$ and $b$ respectively and $\hat{a}_{N N}$ is the asymmetry parameter of the hard subprocess $(a=q$ or $\bar{q}, b=q$ or $\bar{q}$ ). A strong effect is expected in Drell-Yan $p \bar{p}$ collisions where $\hat{a}_{N N}$ is of the order of unity. In $p p$ collisions, the scattering of two identical quarks also has a non vanishing $\hat{a}_{N N}$, which comes from the interference term, but this effect is only of order $1 / N_{\text {color }}$. Nevertheless it should be interesting to detect it, looking at high $x_{T}$ particles or jets, for which the valence + valence mechanism is dominant. The remarkably strong peaking of $\hat{a}_{N N}(\theta)$ at $\theta=\pi / 2$ predicted by (4.15) could be tested.

The quantity $\Delta_{2} G(x)$ exists only for spin $\geqq 1$ projectile or target, in practice for a real or quasi real photon, in the vector dominance model. The linear polarization of both the beam and the target seems extremely difficult.

Acknowledgements. We especially thank M. Fontannaz and D. Schiff for pointing out useful references to us, and C.J. Maxwell for carefully reading this manuscript.

\section{Appendix A}

Calculation of $\Delta_{1} P(z)$

The first $q \rightarrow q G$ vertex which appears in (3.1) is given by

$\langle\mathbf{b}, \beta, \mu ; \mathbf{c}, \gamma, v|V| \mathbf{a}, \alpha, \lambda\rangle=\delta_{\lambda \mu} T_{\beta \alpha}^{\gamma} u^{+}(\mathbf{b}, \lambda) \alpha \cdot \varepsilon^{*}(v) u(\mathbf{a}, \lambda)$,

$\boldsymbol{\varepsilon}(v)$ being the gluon polarization vector. We use the spinorial representation of the Dirac matrices: $\boldsymbol{\alpha}=\left(\begin{array}{cc}\boldsymbol{\sigma} & 0 \\ 0 & -\boldsymbol{\sigma}\end{array}\right), \quad \beta=\left(\begin{array}{ll}0 & 0 \\ 1 & 0\end{array}\right), \quad \gamma_{5}=\left(\begin{array}{cc}-1 & 0 \\ 0 & 1\end{array}\right)$.

The helicity spinors are

$u(\mathbf{p}, \pm)=\left(\begin{array}{l}\sqrt{E \pm p} \\ \sqrt{E \mp p}\end{array}\right) \otimes| \pm\rangle_{\mathbf{p}} \quad(\mathbf{p}=\mathbf{a}$ or $\mathbf{b})$,

where $|\lambda\rangle_{\mathrm{p}}$ is the Pauli spinor of helicity $\sigma \cdot \mathbf{p} / p=\lambda$. We temporarily take the gluon direction as the $z$-axis:

$\mathbf{a} \simeq\left(\mathbf{a}_{T}, a\right) ; \quad \mathbf{b} \simeq\left(\mathbf{a}_{T}, z_{a}\right) ;$

$\mathbf{c} \simeq(\mathbf{0},(1-z) a)$,

$a_{T}$ being related to $k_{T}$ of $(3.1)$ by

$a_{T}=(1-z)^{-1} k_{T}$.

$|\lambda\rangle_{p}$ is obtained from the eigenvector $|\lambda\rangle$ of $\sigma_{z}$ by rotation of axial-vector angle $\boldsymbol{\theta} \cong \hat{z} \times \hat{p}$. To first order in $p_{T} / p$,

$$
\begin{aligned}
|\lambda\rangle_{\mathrm{p}} & \cong \exp \left[\frac{-i}{2 p}(\hat{z} \times \mathbf{p}) \cdot \boldsymbol{\sigma}\right]|\lambda\rangle \\
& \cong\left(1+\frac{1}{2 p} \boldsymbol{\sigma} \cdot \mathbf{p}_{T} \sigma_{z}\right)|\lambda\rangle .
\end{aligned}
$$

Thus, neglecting masses,

$$
\begin{aligned}
& u^{+}(\mathbf{b}, \lambda) \boldsymbol{\alpha} \cdot \varepsilon^{*}(v) u(\mathbf{a}, \lambda)=2 \lambda \sqrt{a b} \\
& \left\langle\lambda\left|\left(1+\frac{1}{2 b} \sigma_{z} \boldsymbol{\sigma} \cdot \mathbf{a}_{T}\right) \boldsymbol{\sigma} \cdot \varepsilon^{*}(v)\left(1+\frac{1}{2 a} \boldsymbol{\sigma} \cdot \mathbf{a}_{T} \sigma_{z}\right)\right| \lambda\right\rangle \\
& =\sqrt{2} \frac{k_{x}-i v k_{y}}{z(1-z)} z^{|\lambda-v|} \quad\left(\lambda= \pm \frac{1}{2}, v= \pm 1\right),
\end{aligned}
$$

where we have used relation (A.3). (To obtain this result, it is advantageous to make use of the $\sigma_{+}$ matrices). Putting the results (A.1) and (A.5) into (3.1) with $\lambda=\mu=-\lambda^{\prime}=-\mu^{\prime}$, we get finally [21]

$\Delta_{1} P(z)=C_{2}(R)\left(\frac{2}{1-z}-2\right) \quad(z<1)$

with

$C_{2}(R)=\sum_{\beta, \gamma}\left|T_{\beta \alpha}^{\gamma}\right|^{2}=\frac{N^{2}-1}{2 N}$.

For $\lambda^{\prime}=\lambda$, we recover the well-known result

$P_{q q}(z)=C_{2}(R) \frac{1+z^{2}}{1-z} \quad(z<1)$.

Expression (A.6) diverges at $z \rightarrow 1$. This divergence is compensated by the renormalizations of the quark propagators (Fig. 3b) which add to (3.1) a counter-term of the form: (infinite constant) $\times \delta(z-1) \times \delta_{\lambda \mu^{\prime}} \delta_{\lambda^{\prime} \mu^{\prime}}$. Due to its particular helicity dependence, the counterterm takes the same value in $P_{q q}(z)$ and $\Delta_{1} P(z)$. It amounts to making the substitution [2]

$\frac{2}{1-z} \rightarrow\left(\frac{2}{1-z}\right)_{+}+\frac{3}{2} \delta(z-1)$. 


\section{Appendix B}

Calculation of $\Delta_{2} P(z)$

The $G \rightarrow G G$ vertex is given by

$$
\begin{aligned}
\langle\mathbf{b}, \beta, \mu ; \mathbf{c}, \gamma, v|V| \mathbf{a}, \alpha, \lambda\rangle \\
=f_{\alpha \beta \gamma}\left[\boldsymbol{\varepsilon}^{*}(\mu) \cdot \boldsymbol{\varepsilon}^{*}(v)(\mathbf{c}-\mathbf{b}) \cdot \boldsymbol{\varepsilon}(\lambda)\right. \\
\left.\quad-\varepsilon^{*}(v) \cdot \varepsilon(\lambda)(\mathbf{a}+\mathbf{c}) \cdot \boldsymbol{\varepsilon}^{*}(\mu)+\boldsymbol{\varepsilon}(\lambda) \cdot \varepsilon^{*}(\mu)(\mathbf{b}+\mathbf{a}) \cdot \varepsilon^{*}(v)\right] \\
=f_{\alpha \beta \gamma} \sqrt{2}\left[k_{x}+i(\lambda-\mu-v) k_{y}\right] \\
\quad\left(-\delta_{\mu,-v}+\frac{1}{z} \delta_{v, \lambda}+\frac{1}{1-z} \delta_{\lambda, \mu}\right) .
\end{aligned}
$$

We are interested in the case $\lambda=\mu$. Using a shorter notation,

$\langle\lambda, \mu|V| \lambda\rangle=f_{\alpha \beta \gamma} \sqrt{2} \frac{k_{x}-i v k_{y}}{z(1-z)} z^{|\lambda-v|,}$

which is very similar to (A.5) expect for the fact that now $|\lambda|=|v|=1$. Putting this result into (3.1) with $\lambda=\mu=-\lambda^{\prime}=-\mu^{\prime}$, we get

$\Delta_{2} P(z)=C_{2}(G)\left(\frac{2}{1-z}-2\right) \quad(z<1)$

with

$$
C_{2}(G)=\sum_{\beta \gamma}\left(f_{\alpha \beta \gamma}\right)^{2}=N \text {. }
$$

The similarity between (B.2) and (A.6) is related to the one between (B.1) and (A.5). Here again we have to regularize by the infinite renormalization counterterm, which is the same as for $P_{G G}(Z)$. We make therefore the substitution [2]

$\frac{2}{1-z} \rightarrow\left(\frac{2}{1-z}\right)_{+}+\left(\frac{11}{6}-\frac{2 T(R)}{3 C_{2}(G)}\right) \delta(z-1)$

\section{Appendix C}

A Naive covariant parton model for polarized quark distributions

Let us suppose that the baryon $B$ is a bound state of a quark and a scalar, isoscalar diquark $d$, the $q-d-B$ vertex being

$g\langle\mu|V| \hat{\lambda}\rangle=g \bar{u}\left(x \mathbf{p}+k_{T}, \mu\right) u(\mathbf{p}, \lambda)$.

Thus, using (A.2) and (A.4),

$\langle+|V|+\rangle=\langle-|V|-\rangle=\left(m_{q}+x m_{B}\right) x^{-1 / 2}$

$\langle+|V|-\rangle=-\langle-|V|+\rangle^{*}=\left(k_{x}-i k_{y}\right) x^{-1 / 2}$.

In the Weisszäcker-Williams approximation [22], the density of quark polarized in the direction $\hat{n}_{q}$ in a baryon polarized in the direction $\hat{n}_{B}$ is given by

$$
\begin{aligned}
d N_{q}= & \left(16 \pi^{3}\right)^{-1} g^{2}\left|\left\langle\hat{n}_{q}|V| \hat{n}_{B}\right\rangle\right|^{2} \\
& \cdot\left(k^{2}-m_{q}^{2}\right)^{-2} x(1-x)^{-1} d x d^{2} \mathbf{k}_{T},
\end{aligned}
$$

where $k$ is the four-momentum of the quark, which is off-mass-shell whereas the spectator diquark is on-shell. We have

$$
\begin{aligned}
& k^{2}=x m_{B}^{2}-\left(k_{T}^{2}+x m_{d}^{2}\right)(1-x)^{-1}, \\
& \left|\left\langle k \hat{n}_{q}|V| \hat{n}_{B}\right\rangle\right|^{2} \\
& \left\{\begin{array}{lll}
=\left(m_{q}+x m_{B}\right)^{2} / x & \text { for } & q_{+}\left(\hat{n}_{B}=\hat{n}_{q}=\hat{z}\right) \\
=k_{T}^{2} / x & \text { for } & q_{-.}\left(\hat{n}_{B}=-\hat{n}_{q}=\hat{z}\right) \\
=\left[\left(m_{q}+x m_{B}\right)^{2}+k_{y}^{2}\right] / x & \text { for } & q_{+\hat{x}}\left(\hat{n}_{B}=\hat{n}_{q}=\hat{x}\right) \\
=k_{x}^{2} / x & \text { for } & q_{-\hat{x}}\left(\hat{n}_{B}=-\hat{n}_{q}=\hat{x}\right) .
\end{array}\right.
\end{aligned}
$$

Integrating over $\mathbf{k}_{T}$, we get

$q_{+}(x)=C_{0}\left(m_{q}+x m_{B}\right)^{2} \int_{0}^{\infty} d k_{T}^{2}(1-x)^{-1}\left[m_{q}^{2}-k^{2}\left(x, k_{T}\right)\right]^{-2}$,

$q_{-}(x)=C_{0} \int_{0}^{\infty} k_{T}^{2} d k_{T}^{2}(1-x)^{-1}\left[m_{q}^{2}-k^{2}\left(x, k_{T}\right)\right]^{-2}$,

with $C_{0}=g^{2} / 16 \pi^{2}$. Furthermore, owing to $\left\langle k_{x}^{2}\right\rangle=$ $\left\langle k_{y}^{2}\right\rangle=\frac{1}{2}\left\langle k_{T}^{2}\right\rangle$,

$q_{-\lambda}(x)=q_{+}(x)+\frac{1}{2} q_{-}(x)$,

$q_{-\lambda}(x)=\frac{1}{2} q_{-}(x)$.

Hence

$\Delta_{1} q(x) \equiv q_{+\dot{x}}(x)-q_{-\dot{x}}(x)=q_{+}(x)$,

which is the result (1.2) quoted in the introduction.

Actually, the integrals (C.5) diverge at large $k_{T}$. To cure this fact, let us replace the quark propagator by a gaussian in $\mathbf{k}_{\mathbf{T}}$ :

$C_{0}\left[m_{q}^{2}-k^{2}\left(x, k_{T}\right)\right]^{-2} \rightarrow C R^{2} \exp \left[R^{2} k^{2}\left(x, k_{T}\right)\right]$.

This takes account (i) of the softness of the hadronic wave function, (ii) of confinement (the quark pole should be absent). We get

$q_{+}(x)=f(x)\left(m_{q}+x m_{B}\right)^{2}=\Delta_{1} q(x)$,

$q_{-}(x)=f(x)(1-x) / R^{2}$,

$f(x)=C \exp \left[x R^{2}\left(m_{B}^{2}-(1-x)^{-1} m_{d}^{2}\right)\right]$.

It is to be noted that the relation (C.7) is independent of the particular choice of transverse momentum cut-of. It seems only from (C.4).

\section{References}

1. R.P. Feynman: Photon-hadron interactions, New York: Benjamin 1972

2. G. Altarelli, G. Parisi: Nucl. Phys. B 126 (1977) 298, and references therein.

3. Yu L. Dokshitzer, D.I. Dyakonov, S.I. Troyan: Phys. Rep. 58 (1980) 270, and references therein

4. See, for instance: a) N.S. Craigie, K. Hidaka, M. Jacob, F.M. Renard: Phys. Rep. 99 (1983) 69; b) K. Hikasa: Phys. Rev. D33 (1986) 3203

5. I. Antoniadis, C. Kounnas: Phys. Rev. D24 (1981) 505 and references therein

6. F. Delduc, M. Gourdin, E.G. Oudrhiri-Safiani: Nucl. Phys. B174 (1980) 157 
7. a) M. Mekhfi, X. Artru: Proc. of the XIXth International Symposium on Multiparticle Dynamics (Arles, France, 1988), D. Schiff, J. Tran Thanh Van (eds) p. 111; Gif-sur-Yvette: Editions Frontières 1988; b) X. Artru, M. Mekhfi, LPTHE 89/11 (Orsay) 1989

8. The decomposition (2.13a) of the density matrix has some similarities with the multipole expansion, (2.32) of [9]. In fact, for spin $\frac{1}{2}$, the spherical tensor operators $T_{0}^{0}, T_{0}^{1}, T_{ \pm 1}^{1}$ are equal to $\mathcal{O}\left(0_{+}\right), \mathcal{O}\left(0_{-}\right)$and $\mathcal{O}( \pm 1)$ respectively, up to numerical factors. In fact, for spin $\frac{1}{2}$, the spherical tensor operators $T_{0}^{0}$, $T_{0}^{1}, T_{+1}^{1}$ are equal to $\mathcal{O}\left(0_{+}\right), \mathcal{O}\left(0_{-}\right)$and $\mathcal{O}( \pm 1)$ respectively, up to numerical factors (or normalization (2.12) is simpler than the normalization (2.33) of Ref. [9]). This is no longer true, however, for higher spin. The multipole expansion is not suited to massless particles of spin $\geqq 1$ because it involves all helicities between $-s$ and $+s$

9. C. Bourrely, E. Leader, J. Soffer: Phys. Rep. 59 (1980) 95

10. We define the helicity states for backward particles by $\mid \mathbf{p}=$ $(0,0,-p) ; \lambda\rangle=e^{-i \pi J_{y}}|p=(0,0,+p) ; \lambda\rangle$

11. These results were reported in [7a]. (3.2a) differs from the corresponding one, (80) of Antoniadis and Kounnas [5]. (3.2b) is in agreement with the kernel of the photon structure function $\mathrm{H}_{7}$ in [6] if one corrects an obvious mistake: their $11 / 6$ term must be multiplied by $\delta(1-x)$. In (17) of [7a], the term in $\delta(z-1)$ of $\Delta^{2} P_{G G}$ (improperly written $\Delta^{2} P_{q q}$ ) must be multiplied by 2

12. M.B. Einhorn, J. Soffer: Nucl. Phys. B274 (1986) 714; E. Richter-Was, J. Swed: Z. Phys. C-Particles and Fields 31 (1986) 253

13. See, for instance, S.J. Parke, T.R. Taylor: Phys. Rev. Lett. 56 (1986) 2459; I.G. Knowles: Cavendish-HEP-88/5

14. This is not really a restriction since the parity violating weak interactions involve only left-handed quarks or right-handed antiquarks so that $\delta_{a}=\delta_{b}=0$

16. See, for instance, (7.4) of [4a]

17. See also [18]. We think, however, that these authors are too pessimistic about the size of the effect. Their estimation of $\Delta_{1} q(x)=g_{1}(x)+g_{2}(x)$ is model dependent

18. K. Hidaka, E. Monsay, D. Sivers: Phys. Rev. D19 (1979) 1503

19. We have made an independent calculation of this result. It agrees with $a_{N N}\left(90^{\circ}\right)=-1 / 11$ quoted in $[18,20]$. For $\theta \neq 0$, our results agrees with (8) of [18] if one changes the sign of their last term in $\delta_{\alpha \beta} / 3 \hat{f} \hat{u}$

20. C.K. Chen: Phys; Rev. Lett. 41 (1978) 1440

21. We have checked this result using a covariant approach.

22. See [2] and references therein. The reference to $P$. Kessler should be corrected: Nuovo Cimento 71 (1960) 809

23. J. Ashman et al. EMC Coll.: Phys. Lett. B206 (1988) 364 